MATHEMATICS OF GRAVITATION

PART II, GRAVITATIONAL WAVE DETECTION

BANACH CENTER PUBLICATIONS, VOLUME 41

INSTITUTE OF MATHEMATICS

POLISH ACADEMY OF SCIENCES

WARSZAWA 1997

\title{
A XYLOPHONE DETECTOR IN SPACE
}

\author{
MASSIMO TINTO \\ Jet Propulsion Laboratory \\ 4800 Oak Grove Drive, Pasadena, CA, U.S.A. \\ E-mail: Massimo.Tinto@jpl.nasa.gov
}

\begin{abstract}
We discuss spacecraft Doppler tracking for detecting gravitational waves in which Doppler data recorded on the ground are linearly combined with Doppler measurements made on board a spacecraft. By using the four-link radio system first proposed by Vessot and Levine [1] we derive a new method for removing from the combined data the frequency fluctuations due to the Earth troposphere, ionosphere, and mechanical vibrations of the antenna on the ground. This method also reduces the frequency fluctuations of the clock on board the spacecraft by several orders of magnitude at selected Fourier components, making Doppler tracking the equivalent of a xylophone detector of gravitational radiation [2]. In the assumption of calibrating the frequency fluctuations induced by the interplanetary plasma, a strain sensitivity equal to $4.7 \times 10^{-18}$ at $10^{-3} \mathrm{~Hz}$ is estimated.

This experimental technique could be extended to other tests of the theory of relativity, and to radio science experiments that rely on high-precision Doppler measurements.
\end{abstract}

1. Introduction. Spacecraft Doppler tracking allows accurate measurements of distances and velocities of objects in the solar system, leading to information on masses and higher order moments of gravity fields of planets, their satellites, and asteroids [3, 4]. Doppler measurements have also been utilized to search for gravitational waves in the millihertz frequency region $[5,6]$, and for placing upper limits on amplitudes of signals characterizing relativistic effects $[7,8]$. However, as we mentioned in our first lecture, Doppler observations suffer from noise sources that can be, at best, only partially reduced or calibrated by implementing specialized and expensive hardware. The fundamental limitation is imposed by the frequency fluctuations inherent in the clocks referencing the microwave system. Current generation Hydrogen masers achieve their best performance at about 1000 seconds integration time with a fractional frequency stability of a few parts

1991 Mathematics Subject Classification: 83C35, 83B05.

This work was performed at the Jet Propulsion Laboratory, California Institute of Technology, under a contract with the National Aeronautics and Space Administration.

The paper is in final form and no version of it will be published elsewhere. 
in $10^{-16}$. This integration time is also comparable to the propagation time to spacecraft in the outer solar system.

The frequency fluctuations induced by the intervening media have severely limited the sensitivities of these experiments. Among all the propagation noise sources, the troposphere is the largest and the hardest to calibrate to a reasonably low level. Its frequency fluctuations have been estimated to be as large as $10^{-13}$ at 1000 seconds integration time [9].

In order to systematically remove the frequency fluctuations due to the troposphere in the Doppler data, it was pointed out by Vessot and Levine [1] and Smarr et al. [10] that by adding to the spacecraft payload a highly stable frequency standard, a Doppler read-out system, and by utilizing a transponder at the ground antenna, one could make Doppler one-way (Earth-to-spacecraft, spacecraft-to-Earth) as well as twoway (spacecraft-Earth-spacecraft, Earth-spacecraft-Earth) measurements. This way of operation makes the Doppler link totally symmetric and allows the complete removal of the frequency fluctuations due to the Earth troposphere, ionosphere, and mechanical vibrations of the ground antenna by properly combining the Doppler data recorded on the ground with the data measured on the spacecraft. Their proposed scheme relied on the possibility of flying a Hydrogen maser on a dedicated mission. Although current designs of Hydrogen masers have demanding requirements in mass and power consumption, it seems very likely that by the beginning of the next century new space-qualified atomic clocks, with frequency stability of a few parts in $10^{-16}$ at 1000 seconds integration time, will be available. They would provide a sensitivity gain of almost a factor of one thousand with respect to the best performance crystal-driven oscillators. Although this clearly would imply a great improvement in the technology of space born clocks, it would not allow us to reach a Doppler sensitivity better than a few parts $10^{-16}$. This would be only a factor of five or ten better than the Doppler sensitivity expected to be achieved on the future Cassini project, a NASA mission to Saturn, which will take advantage of a high radio frequency link $(32 \mathrm{GHz})$ in order to minimize the plasma noise, and will use a purposely built water vapor radiometer for calibrating up to ninety five percent the frequency fluctuations due to the troposphere [11].

In this paper we adopt the radio link configuration first envisioned by Vessot and Levine [1], but we combine the Doppler responses measured on board the spacecraft and on the ground in a different way, as it will be shown in the following sections. Furthermore our technique allows us to reduce by several orders of magnitude, at selected Fourier components, the noise due to the clock on board the spacecraft [2]. This experimental approach could also be extended to other tests of the relativistic theory of gravity and to radio science experiments that rely on high-precision Doppler measurements.

2. Doppler tracking as a xylophone detector. In Doppler tracking experiments a distant interplanetary spacecraft is monitored from Earth through a radio link, and the Earth and the spacecraft act as free-falling test particles. In a one-way operation a radio signal of nominal frequency $\bar{\nu}_{0}$ referenced to an onboard clock is transmitted to Earth, where it is compared to a signal referenced to a highly stable clock. In a two-way operation 
instead a radio signal of frequency $\nu_{0}$ is transmitted to the spacecraft, and coherently transponded back to Earth. In both configurations relative frequency changes $\Delta \nu / \nu_{0}$ as functions of time are measured, and the physical effects the experimenter is trying to observe appear in the Doppler observable as small frequency changes of well defined time signature $[3,4,5,7]$. In searches for gravitational waves only two-way data have been used so far due to lack of space qualified, high performance, clocks. In what follows we will not make any assumption on the frequency stability of the onboard oscillator and return to this point later in the paper.

If a Doppler readout system is added to the spacecraft radio instrumentation, and a transponder is installed at the ground station, one-way as well as two-way Doppler data can also be recorded on board the spacecraft [1]. If we assume the Earth clock and the onboard clock to be synchronized, then the one-way and two-way Doppler data measured at time $t$ on the Earth $\left(E_{1}(t), E_{2}(t)\right.$ respectively), and the one-way and two-way Doppler measured at the same time $t$ on the spacecraft $\left(S_{1}(t), S_{2}(t)\right)$, are given by the following rather complete expressions [2]

$$
\begin{gathered}
E_{1}(t)=\frac{(1-\mu)}{2}[h(t-(1+\mu) L)-h(t)]+C_{s c}(t-L)-C_{E}(t)+ \\
\quad+T(t)+B(t-L)+A_{s c}(t-L)+E L_{E_{1}}(t)+P_{E_{1}}(t), \\
E_{2}(t)=-\frac{(1-\mu)}{2} h(t)-\mu h(t-(1+\mu) L)+\frac{(1+\mu)}{2} h(t-2 L)+ \\
+C_{E}(t-2 L)-C_{E}(t)+2 B(t-L)+T(t-2 L)+T(t)+ \\
+A_{E}(t-2 L)+A_{s c}(t-L)+T R_{s c}(t-L)+E L_{E_{2}}(t)+P_{E_{2}}(t), \\
S_{1}(t)=\frac{(1+\mu)}{2}[h(t-L)-h(t-\mu L)]+C_{E}(t-L)-C_{s c}(t)+ \\
\quad+T(t-L)+B(t)+A_{E}(t-L)+E L_{S_{1}}(t)+P_{S_{1}}(t), \\
\quad(1+\mu) \\
\quad h(t-\mu L)+\mu h(t-L)+\frac{(1-\mu)}{2} h(t-2 L-\mu L)+ \\
\quad+C_{s c}(t-2 L)-C_{s c}(t)+2 T(t-L)+B(t-2 L)+B(t)+ \\
+A_{s c}(t-2 L)+A_{E}(t-L)+T R_{E}(t-L)+E L_{S_{2}}(t)+P_{S_{2}}(t),
\end{gathered}
$$

where $h(t)$ is equal to

$$
h(t)=h_{+}(t) \cos (2 \phi)+h_{\times}(t) \sin (2 \phi),
$$

and is the gravitational wave signal. In Eqs. (1-4) $\mu$ is the cosine of the angle between the direction of propagation of the wave and the line of sight to the spacecraft, $h_{+}(t)$, $h_{\times}(t)$ are the wave's two independent amplitudes referenced to a given set of axes defined in the plane of the wave, $\phi$ is the polar angle describing the projection of the direction to the spacecraft in the plane of the wave, and $L$ is the distance to the spacecraft $[2,5]$ (units in which the speed of light $c=1$ ).

In Eqs. (1-4) $T R_{s c}(t)$ and $T R_{E}(t)$ represent the noise due to the transponder on board and on the ground respectively, $E L_{E_{1}}(t), E L_{E_{2}}(t), E L_{S_{1}}(t)$, and $E L_{S_{2}}(t)$ the noises from the electronics at the ground station and on the spacecraft in the one-way and two-way data, and $P_{E_{1}}(t), P_{E_{2}}(t), P_{S_{1}}(t)$, and $P_{S_{2}}(t)$ the frequency fluctuations due 
to the interplanetary plasma. The plasma noise can be entirely calibrated by using dual frequencies [11]. The Doppler data $S_{1}(t)$ and $S_{2}(t)$ are then time tagged, and telemetered back to Earth in real time or at a later time during the mission.

It is important to note the characteristic time signatures of the frequency fluctuations due to the Earth clock $\left(C_{E}\right)$, the onboard clock $\left(C_{s c}\right)$, the spacecraft buffeting $(B)$, the troposphere, ionosphere, and mechanical vibrations of the ground antenna, $(T)$, the Earth transmitter, $\left(A_{E}\right)$, and the onboard amplifier $\left(A_{s c}\right)[2,10]$. It was first pointed out by Vessot and Levine [1] that by properly combining some of the four Doppler data streams it was possible to calibrate the frequency fluctuations of the troposphere, ionosphere, and ground antenna noise, $T(t)$. Their pioneering work, however, left open the question on whether there existed some other, perhaps more complicated, linear combinations of the data that would further improve the sensitivity of Doppler tracking. In what follows we answer this question, and derive a method that allows us to uniquely identify an optimal way of combining the data.

Let $\widetilde{E_{1}}(f)$ be the Fourier transform of the time series $E_{1}(t)$

$$
\widetilde{E_{1}}(f) \equiv \int_{-\infty}^{+\infty} E_{1}(t) e^{2 \pi i f t} d t
$$

and similarly let us denote by $\widetilde{E_{2}}(f), \widetilde{S_{1}}(f), \widetilde{S_{2}}(f)$ the Fourier transforms of $E_{2}(t), S_{1}(t)$, and $S_{2}(t)$ respectively. The most general linear combination of the four Doppler data given in Eqs. $(1,2,3,4)$, can be written in the Fourier domain as follows:

$$
\widetilde{y}(f) \equiv a(f, L) \widetilde{E_{1}}(f)+b(f, L) \widetilde{E_{2}}(f)+c(f, L) \widetilde{S_{1}}(f)+d(f, L) \widetilde{S_{2}}(f),
$$

where the coefficients $a, b, c, d$ are for the moment arbitrary functions of $f$ and $L$. If we substitute in Eq. (6) the Fourier transforms of Eqs. (1, 2, 3, 4) we deduce the following expression

$$
\begin{aligned}
\widetilde{y}(f)= & \left\{a \frac{(1-\mu)}{2}\left[e^{2 \pi i f(1+\mu) L}-1\right]+b\left[\frac{(\mu-1)}{2}-\mu e^{2 \pi i f(1+\mu) L}+\frac{(1+\mu)}{2} e^{4 \pi i f L}\right]\right. \\
& +c \frac{(1+\mu)}{2}\left[e^{2 \pi i f L}-e^{2 \pi i f \mu L}\right]+d\left[-\frac{(\mu+1)}{2} e^{2 \pi i f \mu L}+\mu e^{2 \pi i f L}\right. \\
& \left.\left.+\frac{(1-\mu)}{2} e^{2 \pi i f(2+\mu) L}\right]\right\} \widetilde{h}(f) \\
& +\widetilde{C_{E}}(f)\left[-a+b\left(e^{4 \pi i f L}-1\right)+c e^{2 \pi i f L}\right] \\
& +\widetilde{C_{s c}}(f)\left[a e^{2 \pi i f L}-c+d\left(e^{4 \pi i f L}-1\right)\right] \\
& +\widetilde{T}(f)\left[a+b\left(e^{4 \pi i f L}+1\right)+c e^{2 \pi i f L}+2 d e^{2 \pi i f L}\right] \\
& +\widetilde{B}(f)\left[a e^{2 \pi i f L}+2 b e^{2 \pi i f L}+c+d\left(e^{4 \pi i f L}+1\right)\right] \\
& +\widetilde{A_{E}}(f)\left[b e^{4 \pi i f L}+c e^{2 \pi i f L}+d e^{2 \pi i f L}\right] \\
& +\widetilde{A_{s c}}(f)\left[a e^{2 \pi i f L}+b e^{2 \pi i f L}+d e^{4 \pi i f L}\right] \\
& +a\left[\widetilde{E L_{E_{1}}}(f)+\widetilde{P_{E_{1}}}(f)\right]+b\left[\widetilde{T R_{s c}}(f) e^{2 \pi i f L}+\widetilde{E L_{E_{2}}}(f)+\widetilde{P_{E_{2}}}(f)\right] \\
& +c\left[\widetilde{E L_{S_{1}}}(f)+\widetilde{P_{S_{1}}}(f)\right]+d\left[\widetilde{T R_{E}}(f) e^{2 \pi i f L}+\widetilde{E L_{S_{2}}}(f)+\widetilde{P_{S_{2}}}(f)\right] .
\end{aligned}
$$


The four coefficients $a, b, c, d$, can be determined by requiring the transfer functions of the random processes $\widetilde{C_{E}}(f), \widetilde{C_{s c}}(f), \widetilde{T}(f), \widetilde{B}(f), \widetilde{A_{E}}(f), \widehat{A_{s c}}(f)$ in Eq. (7) to be simultaneously equal to zero, and by further checking that each solution gives a non-zero gravitational wave signal in the corresponding combined data. This condition implies that $a, b, c, d$ must satisfy a homogeneous linear system of six equations in four unknowns. We calculated the rank of the $(6 \times 4)$ matrix associated with this linear system by using the algebraic computer language Mathematica, and we found it to be equal to two. The corresponding solution can be written in the following way

$$
\begin{aligned}
& a(f, L)=c(f, L) e^{-2 \pi i f L}-d(f, L)\left[e^{2 \pi i f L}-e^{-2 \pi i f L}\right] \\
& b(f, L)=-c(f, L) e^{-2 \pi i f L}-d(f, L) e^{-2 \pi i f L},
\end{aligned}
$$

where $c$ and $d$ can be any arbitrary complex functions not simultaneously equal to zero. If we substitute Eq. (8) into Eq. (7) however, we find that also the gravitational wave signal, in the combined Doppler data, vanishes. In other words, any linear combination of the four Doppler data that does not contain any clocks, troposphere, ionosphere, mechanical vibrations of the ground antenna, buffeting of the spacecraft, and transmitters noise, has also a null response to a gravitational wave pulse. These results imply that, at any Fourier frequency $f$, we can remove only one of the considered noise sources. Among all the noise sources affecting spacecraft Doppler tracking, the frequency fluctuations due to the troposphere, ionosphere, and mechanical vibrations of the ground antenna, $\widetilde{T}(f)$, are the largest. If we choose $a, b, c, d$ in such a way that the transfer function of $\widetilde{T}(f)$ in the combined data is equal to zero, from Eq. (7) we find that $a, b, c, d$, must satisfy the following condition

$$
a(f, L)=-b(f, L)\left[e^{4 \pi i f L}+1\right]-c(f, L) e^{2 \pi i f L}-2 d(f, L) e^{2 \pi i f L} .
$$

Since $b, c, d$ can not be equal to zero simultaneously, we will choose $c$ to be equal to $1 / 2$, and $b, d$ to be equal to zero. In other words we will consider only linear combinations of one-way Doppler data. Note that with this choice we eliminate from the combined data $y(t)$ the frequency fluctuations due to the transponders and the interplanetary plasma that affect the two-way Doppler data. These considerations imply the following expression for $\widetilde{y}(f)$

$$
\widetilde{y}(f)=\frac{1}{2}\left[\widetilde{S_{1}}(f)-\widetilde{E_{1}}(f) e^{2 \pi i f L}\right] .
$$

If we substitute the Fourier transforms of Eqs. (1,3) into Eq. (10) we get

$$
\begin{aligned}
\widetilde{y}(f)= & \frac{e^{2 \pi i f L}}{2}\left[1-\frac{(1+\mu)}{2} e^{2 \pi i f(\mu-1) L}-\frac{(1-\mu)}{2} e^{2 \pi i f(\mu+1) L}\right] \widetilde{h}(f) \\
& +\widetilde{C_{E}}(f) e^{2 \pi i f L}-\frac{1}{2} \widetilde{C_{s c}}(f)\left[e^{4 \pi i f L}+1\right]+\frac{1}{2} \widetilde{B}(f)\left[1-e^{4 \pi i f L}\right] \\
& +\frac{e^{2 \pi i f L}}{2}\left[\widetilde{A_{E}}(f)-\widetilde{A_{s c}}(f) e^{2 \pi i f L}\right]+\frac{1}{2}\left[\widetilde{P_{S_{1}}}(f)-\widetilde{P_{E_{1}}}(f) e^{2 \pi i f L}\right] \\
& +\frac{1}{2}\left[\widetilde{E L_{S_{1}}}(f)-\widetilde{E L_{E_{1}}}(f) e^{2 \pi i f L}\right] .
\end{aligned}
$$

Eq. (11) shows that the transfer functions of the noise of the onboard clock, $\widetilde{C_{s c}}(f)$, and of buffeting $\widetilde{B}(f)$, can in principle be set to zero (not simultaneously) at specific Fourier 
frequencies. In searches for gravitational wave pulses it has been shown [2] that one can reduce by several orders of magnitudes the noise of the onboard clock at the nulls of its transfer function without removing the gravitational wave signal.

3. Expected xylophone sensitivities. In what follows we provide the expression for the noise in $y(t)$ at the xylophone frequencies.

Let $\delta$ be the time interval over which a Doppler tracking search for gravitational waves is performed. The corresponding frequency resolution $\Delta f$ of the data is equal to $1 / \delta$. This implies that the fluctuations of the clock on board can be minimized at the following frequencies

$$
f_{k}=\frac{(2 k-1)}{4 L} \pm \frac{\Delta f}{2} ; \quad k=1,2,3, \ldots
$$

We should point out, however, that these resonant frequencies in general will not be constant, since the distance to the spacecraft will change over a time interval of forty days. As an example, however, let us assume again $L=1 \mathrm{AU}, \delta=40$ days, and $f=5 \times 10^{-4}$ $\mathrm{Hz}$. The variation in spacecraft distance corresponding to a frequency change equal to the resolution bin width $\left(3 \times 10^{-7} \mathrm{~Hz}\right)$ is equal to $1.0 \times 10^{5} \mathrm{Km}$. Trajectory configurations fulfilling a requirement compatible to the one just derived have been observed during past spacecraft missions [9], and therefore we do not expect this to be a limiting factor. At these frequencies, and to first order in $\Delta f L$, the Doppler response $\widetilde{y}\left(f_{k}\right)$ is equal to

$$
\begin{aligned}
\widetilde{y}\left(f_{k}\right) \approx & \frac{i}{2}(-1)^{k}\left[-1+i \mu e^{\frac{\pi}{2} i \mu(2 k-1)}(-1)^{k}\right] \widetilde{h}(f) \pm(\pi i \Delta f L) \widetilde{C_{s c}}\left(f_{k}\right) \\
& \left.+i(-1)^{k+1} \widetilde{C_{E}}\left(f_{k}\right)+\widetilde{B}\left(f_{k}\right)+\frac{1}{2} \widetilde{E L_{S_{1}}}\left(f_{k}\right)-i \widetilde{E L_{E_{1}}}\left(f_{k}\right)(-1)^{k+1}\right] \\
& \left.+\frac{1}{2}\left[\widetilde{P_{S_{1}}}\left(f_{k}\right)-i \widetilde{P_{E_{1}}}\left(f_{k}\right)(-1)^{k+1}\right]+\frac{1}{2} \widetilde{\left[A_{s c}\right.}\left(f_{k}\right)+i(-1)^{k+1} \widetilde{A_{E}}\left(f_{k}\right)\right]
\end{aligned}
$$

For a typical gravitational wave experiment, $\delta=40$ days, and $\Delta f=3.0 \times 10^{-7}$. Therefore the frequency fluctuations of a clock on board a spacecraft that is out to $1 \mathrm{AU}$ are reduced at the xylophone frequencies by the following amount:

$$
\frac{\pi \Delta f L}{c}=4.7 \times 10^{-4}
$$

From Eq. (13) we can estimate the expected root-mean-squared (r.m.s.) noise level $\sigma\left(f_{k}\right)$ of the frequency fluctuations in the bins of width $\Delta f$, around the frequencies $f_{k}(k=$ $1,2,3, \ldots$.$) . This is given by the following expression$

$$
\sigma\left(f_{k}\right)=\left[S_{y}\left(f_{k}\right) \Delta f\right]^{1 / 2}, k=1,2,3, \ldots,
$$

where $S_{y}\left(f_{k}\right)$ is the one-sided power spectral density of the noise sources in the Doppler response $y(t)$ at the frequency $f_{k}$. In what follows we will assume that the random processes representing these noises are uncorrelated with each other, and their one-sided power spectral densities are as given in [2]. In that reference a frequency stability of $1.0 \times$ $10^{-16}$ at 1000 seconds integration time for the clock at the ground station was assumed. Although this is a factor of four better than what has been measured so far [11], it seems very likely that by the beginning of next century such a sensitivity can be achieved. 
As far as the remaining sensitivity figures used in our estimate are concerned, they were obtained from the Riley et al. report [11]. This document is a summary of a detailed study, performed jointly by scientists and engineers of NASA's Jet Propulsion Laboratory and the Italian Space Agency (ASI) Alenia Spazio, for assessing the magnitude and spectral characteristics of the noise sources that will determine the Doppler sensitivity of the future gravitational wave experiment on the Cassini mission.

If dual radio frequencies in the uplink and downlink are used, then the frequency fluctuations due to the interplanetary plasma can be entirely removed [11]. We will refer to this configuration as MODE I. If only one frequency is adopted instead, which we will assume to be Ka-Band $(32 \mathrm{GHz})$, we will refer to this configuration as MODE II. Ka-Band is planned to be used on most of the forthcoming NASA missions, and will be implemented on the ground antennas of the Deep Space Network (DSN) by the year 1999 for the Cassini mission.

The r.m.s. $\sigma\left(f_{k}\right)$ of the noise as a function of the frequencies $f_{k}(k=1,2,3, \ldots$.$) ,$ assuming that an interplanetary spacecraft is out to a distance $L=1.0$ AU has been estimated in reference [2]. For this configuration the fundamental frequency of the xylophone (Eq. (11)) is equal to $5.0 \times 10^{-4} \mathrm{~Hz}$. A complete analysis covering configurations with spacecraft at several other distances is also given in [2].

The MODE I configuration is represented in reference [2] by two curves, depending on whether an atomic clock or a crystal Ultra Stable Oscillator (USO) is operated on board the spacecraft. Sensitivity curves for the MODE II configuration are also included in that reference, again with an atomic clock on board or a USO. The best sensitivity is achieved in the MODE I configuration, regardless of whether an atomic clock or a USO is operated on board the spacecraft. This is because the amplitude of the noise of the clock on board is reduced by a factor $\pi \Delta f L / c=4.7 \times 10^{-4}$ at the xylophone frequencies. At $f=10^{-3}$ $\mathrm{Hz}$ the corresponding r.m.s. noise level is equal to $4.7 \times 10^{-18}$, and it increases to a value of $5.7 \times 10^{-18}$ at $f=10^{-2} \mathrm{~Hz}$. As far as the MODE II configuration is concerned, the r.m.s. noise level is equal to $7.9 \times 10^{-18}$ at $f=10^{-3} \mathrm{~Hz}$, while it decreases to $6.3 \times 10^{-18}$ at $f=10^{-2} \mathrm{~Hz}$. This is due to the fact that the one-sided power spectral density of the fractional frequency fluctuations due to the interplanetary plasma decays as $f^{-2 / 3}$.

4. Conclusions. We have discussed a method for significantly increasing the sensitivity of Doppler tracking experiments aimed at the detection of gravitational waves. The main result of our analysis, deduced in Eq. (13), shows that by flying a frequency reference and by adding a Doppler extractor on board the spacecraft and a transponder at the DSN antenna, we can achieve at selected Fourier components a strain sensitivity of $4.7 \times 10^{-18}$. This sensitivity figure is obtained by completely removing the frequency fluctuations due to the interplanetary plasma, at a Fourier frequency equal to $10^{-3} \mathrm{~Hz}$. Our method relies on a properly chosen linear combination of the one-way Doppler data recorded on board with those measured on the ground. It allows us to remove entirely the frequency fluctuations due to the troposphere, ionosphere, and antenna mechanical, and for a spacecraft that is tracked for forty days out to $1 \mathrm{AU}$ it reduces by almost four orders of magnitude the noise due to the on board clock. 
The experimental technique presented in this paper can be extended to a configuration with two spacecraft tracking each other through a microwave or a laser link. Future space-based laser interferometric detectors of gravitational waves [12], for instance, could implement this technique as a backup option, if failure of some of their components would make the normal interferometric operation impossible.

As a final note, a method similar to the one presented can be used in all those radio science experiments in which one-way and two-way spacecraft Doppler measurements are used as primary data set. We will analyze the implications of the sensitivity improvements that this technique will provide for direct measurements of quantities such as the gravitational red shift, possible anisotropy in the velocity of light, the Parameterized Post-Newtonian parameters, the deflection and time delay by the sun in radio signals, and occultation experiments.

\section{References}

[1] R. F. C. Vessot and M. W. Levine, Gen. Relativ. Gravit. 10, 181 (1979).

[2] M. Tinto, Phys. Rev. D. May 15, 1996.

[3] J. D. Anderson et al., Science, 207, pp. 449-453,1980.

[4] J. D. Anderson et al., Icarus, 71, pp. 337-349, 1986.

[5] F. B. Estabrook and H. D. Wahlquist, Gen. Rel. Grav. , 6 439, 1975.

[6] J. W. Armstrong, R. Woo and F. B. Estabrook, Ap. J., 30 574, 1979.

[7] I. I. Shapiro et al., Journal of Geophysical Research, 82, pp. 4329-4334, 1977.

[8] J. D. Anderson et al., Astronautica, 5, pp. 43-61, 1978.

[9] J. W. Armstrong, In: Gravitational Wave Data Analysis, ed. Schutz, B. F., p. 153 (1989), (Dordrecht, Kluwer).

[10] L. L. Smarr, R. F. C. Vessot, C. A. Lundquist, R. Decher, and T. Piran, Gen. Relativ. Gravit. 15, 2 (1983).

[11] A. L. Riley, D. Antsos, J. W. Armstrong, P. Kinman, H. D. Wahlquist, B. Bertotti, G. Comoretto, B. Pernice, G. Carnicella, and R. Giordani, Jet Propulsion Laboratory Report, Pasadena, California, January 22, (1990).

[12] LISA: Laser Interferometer Space Antenna for the detection and observation of gravitational waves. A Cornerstone Project in ESA's long term space science program Horizon 2000 Plus, Pre-Phase A Report, MPQ 208, (December 1995). 